\title{
Imaging Shape-Dependent Corrosion Behavior of Pt Nanoparticles over Extended Time Using a Liquid Flow Cell and TEM
}

\author{
Jianbo Wu, ${ }^{1,2,3}$ Wenpei Gao, ${ }^{1,2}$ Hong Yang, ${ }^{3}$ and Jian-Min Zuo ${ }^{1,2}$ \\ ${ }^{1}$ Dept of Materials Science and Engineering, University of Illinois, Urbana-Champaign, IL 61801 \\ ${ }^{2}$ Frederick Seitz Materials Research Laboratory, University of Illinois, Urbana-Champaign, IL 61801 \\ ${ }^{3}$ Dept of Chemical and Biomolecular Engineering, University of Illinois, Urbana-Champaign, IL 61801
}

Imaging liquids at nanoscale, or atomic resolution, inside a transmission electron microscope (TEM) holds great promise for the study of liquid phase reactions, including electrochemistry. A capsulated liquid cell with thin $\mathrm{Si}_{3} \mathrm{~N}_{4}$ windows is used to hold a ultra-thin liquid layer (less than $500 \mathrm{~nm}$ thick) $[1,2]$. In a static liquid cell, all the reactants, capping agents and solution are pre-mixed in the cell and the structural evolution is observed and captured after introduction of the cell into TEM. This places a severe limit on the type of reactions that can be studied. The reaction cannot be too slow or too fast for two major reasons: 1) loading and introduction of a liquid cell take multiple steps and each takes significant amount of time to complete, and 2) the reaction should be stable under TEM observation while prolonged electron beam irradiation can alter solution chemistry. To follow the entire reaction process, long exposure time is necessary, especially in a slow reaction. The involved electron beam effects reported include heating, charging and production of free radicals [3].

Here we report a successful observation of the corrosion behavior on Pt nanoparticles over the extended time of 60 minutes. The experiment is enabled using a modified fluid liquid cell with two outlets for liquid flow (Hummingbird Scientific Liquid Flow TEM Holder). This setup enables the study of rapid or extremely slow reaction by pumping the reactants into the liquid cell, which trigger the reaction under TEM observation. Moreover, by pumping fresh chemicals into the cell continuously, irradiation effect could be minimized and the environment inside the liquid cell is close to the real reaction condition. Various shapes of Pt nanoparticles are observed for a study of shape dependent corrosion behavior, which occurs once the $\mathrm{HAuCl}_{4}$ solution is injected. By observing the well-defined icosahedron and cube nanoparticles prepared beforehand [4-6], shape-dependent etching behaviors can be monitored during the corrosion process. By pumping the oxidant agent $\left[\mathrm{AuCl}_{4}\right]^{-}$ion into the liquid cell via syringe pump with precise control of several $\mathrm{uL} / \mathrm{sec}$, the kinetics of Pt surface etching could be studied.

Figure 1 shows the morphological changes of one icosahedral and one cubic nanoparticles of platinum within one hour. The images were recorded using a Gatan Ultrascan CCD camera installed on the JEOL2100 TEM with a LaB6 emitter at $200 \mathrm{kV}$ and at 100k magnification. The first image was taken after the $\left[\mathrm{AuCl}_{4}\right]^{-}$solution was pumped into the cell for $10 \mathrm{~min}$ and accounted as $0 \mathrm{~min}$. The icosahedrons started to change on the corner, which has a curved surface. After measuring the distance between two corners, it shrunk from ca. $40 \mathrm{~nm}$ to $32 \mathrm{~nm}$. The corner of cube, however, is stable during the initial stage of corrosion. And then, it started to be corroded at ca. 12 min after that of icosahedron did while the corner of icosahedron has already been blended to be spherical. The $\{100\}$ surface of cubes occurs to be etched by $\left[\mathrm{AuCl}_{4}\right]^{-}$and became to a slightly concave cube along with truncated corner after $18 \mathrm{~min}$. In the meanwhile, the entire icosahedron was etched continually, but the corrosion rate on icosahedron is slower than that on cube. After ca. $60 \mathrm{~min}$, the cube was completed etched and the icosahedral nanoparticles shrunk into a 10-nm nanoparticles. The bright field TEM images were taken 
every three minutes using an exposure time of $1 \mathrm{sec}$. The entitle corrosion process was also captured on video at $0.1 \mathrm{~s}$ intervals.

In summary, we show an in-situ observation of the corrosion behavior on Pt surface of cube and icosahedron in a fluid flow cell via TEM. The liquid flow and careful observation demonstrate that the corrosion is sensitive to surface. The etching rate is highly depending on the surface geometry. This in-situ corrosion observation can provide and establish a valuable database to screen out a stable metal surface, which is very important to the corrosion industry and catalysis.

\section{References:}

[1] M. J. Williamson, R. M. Tromp, P. M. Vereecken, R. Hull, F. M. Ross, Nature Materials 2532 (2003)

[2] H. Zheng, R. K. Smith, Y. W. Jun, C. Kisielowski, U. Dahmen, P. A. Alivisatos, Science 324, 1309 (2009)

[3] J. M. Grogan, N. M. Schneider, F. M. Ross, H. H. Bau, Nano Lett. 14, 359 (2014)

[4] W. Zhou, J. B. Wu, H. Yang, Nano Lett. 13, 2870 (2013)

[5] J. B. Wu, L. Qi, H. J. You, A. Gross, J. Li, H. Yang, J. Am. Chem. Soc., 134, 11880 (2012)

[6] J. B. Wu, A. Gross, H. Yang, Nano Lett., 11, 798 (2011)

[7] The work reported here is supported by the NSF MRI Grant NSF DMR 12-29454 and NSF DMR 0449790.

Jianbo Wu is partially supported by DOE BES under contract DEFG02-01ER45923.

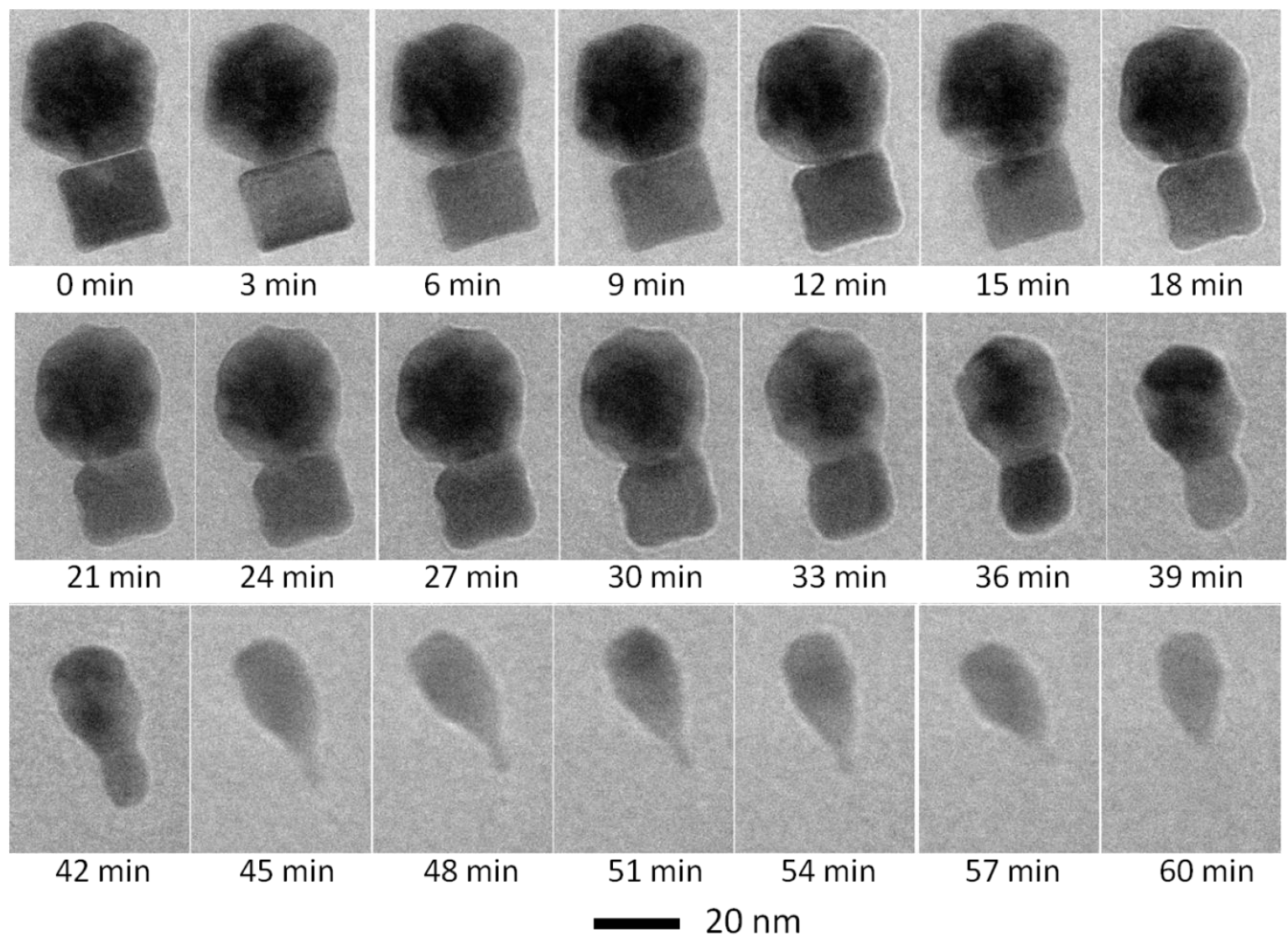

Figure 1 TEM images recording the morphological changes of $\mathrm{Pt}$ icosahedron and cube in the presence of [AuCl4] $]^{-}$solution within $60 \mathrm{~min}$ (for details, see text). 